\title{
Cutoff Values of Serum IgG4 and Histopathological IgG4+ Plasma Cells for Diagnosis of Patients with IgG4-Related Disease
}

\author{
Yasufumi Masaki, ${ }^{1}$ Nozomu Kurose, ${ }^{2}$ Motohisa Yamamoto, ${ }^{3}$ \\ Hiroki Takahashi, ${ }^{3}$ Takako Saeki, ${ }^{4}$ Atsushi Azumi, ${ }^{5}$ Shinji Nakada, ${ }^{6}$ Shoko Matsui, ${ }^{7}$ \\ Tomoki Origuchi, ${ }^{8}$ Susumu Nishiyama, ${ }^{9}$ Kazunori Yamada, ${ }^{10}$ Mitsuhiro Kawano, ${ }^{10}$ \\ Akira Hirabayashi, ${ }^{11}$ Keita Fujikawa, ${ }^{12}$ Tomoko Sugiura, ${ }^{13}$ Masanobu Horikoshi, ${ }^{14}$ \\ Naoto Umeda, ${ }^{14}$ Hiroshi Minato, ${ }^{2}$ Takuji Nakamura, ${ }^{1}$ Haruka Iwao, ${ }^{1}$ Akio Nakajima, ${ }^{1}$ \\ Miyuki Miki, ${ }^{1}$ Tomoyuki Sakai, ${ }^{1}$ Toshioki Sawaki, ${ }^{1}$ Takafumi Kawanami, ${ }^{1}$ \\ Yoshimasa Fujita, ${ }^{1}$ Masao Tanaka, ${ }^{1}$ Toshihiro Fukushima, ${ }^{1}$ Katumi Eguchi, ${ }^{8}$ \\ Susumu Sugai, ${ }^{1}$ and Hisanori Umehara ${ }^{1}$ \\ ${ }^{1}$ Hematology and Immunology, Kanazawa Medical University, 1-1 Daigaku, Uchinada, Kahoku-gun, Ishikawa 920-0293, Japan \\ 2 Department of Pathology and Laboratory Medicine, Kanazawa Medical University, 1-1 Daigaku, Uchinada, Kahoku-gun, \\ Ishikawa 920-0293, Japan \\ ${ }^{3}$ First Department of Internal Medicine, School of Medicine, Sapporo Medical University, S1 W17 Chuo-ku, Sapporo, \\ Hokkaido 060-8556, Japan \\ ${ }^{4}$ Department of Internal Medicine, Nagaoka Red Cross Hospital, Sensyu-2 297-1, Nagaoka-shi, Niigata 940-2085, Japan \\ ${ }^{5}$ Ophthalmology Department, Kobe Kaisei Hospital, Shinohara Kitamachi 3-11-15, Nada Ku, Kobe City, Hyogo 657-0068, Japan \\ ${ }^{6}$ Department of Japanese Oriental Medicine, University of Toyama, 2630 Sugitani, Toyama city, Toyama 930-0194, Japan \\ ${ }^{7}$ First Department of Internal Medicine, University of Toyama, 2630 Sugitani, Toyama city, Toyama 930-0194, Japan \\ ${ }^{8}$ Nagasaki Graduate School of Health Sciences, 1-7-1 Sakamoto, Nagasaki city, Nagasaki 852-8501, Japan \\ ${ }^{9}$ Rheumatic Disease Center, Kurashiki Medical Center, 250 Bakuro-chou, Kurashiki, Okayama 710-8522, Japan \\ ${ }^{10}$ Division of Rheumatology, Department of Internal Medicine, Kanazawa University, 13-1 Takara-machi, Kanazawa, \\ Ishikawa 920-8641, Japan \\ ${ }^{11}$ Hiroshima Bay Clinic, 2-2-19 Yanoshin-machi, Aki-ku, Hiroshima city, Hiroshima 736-0084, Japan \\ ${ }^{12}$ Department of Internal Medicine, Isahaya General Hospital, 24-1 Eishohigasi-macki, Isahaya, Nagasaki 854-8501, Japan \\ ${ }^{13}$ Sugiura Clinic, 2-8-3 Imaichi-chou, Kitashin-machi, Izumo, Shimane 693-0002, Japan \\ ${ }^{14}$ Department of Rheumatology, University of Tsukuba, 2-1-1 Amakubo, Tsukuba, Ibaraki 305-8576, Japan
}

Correspondence should be addressed to Yasufumi Masaki, yasum@kanazawa-med.ac.jp

Received 1 December 2011; Revised 29 February 2012; Accepted 29 February 2012

Academic Editor: John H. Stone

Copyright ( $) 2012$ Yasufumi Masaki et al. This is an open access article distributed under the Creative Commons Attribution License, which permits unrestricted use, distribution, and reproduction in any medium, provided the original work is properly cited.

IgG4-related disease is a new disease classification established in Japan in the 21st century. Patients with IgG4-related disease display hyper-IgG4-gammaglobulinemia, massive infiltration of IgG4+ plasma cells into tissue, and good response to glucocorticoids. Since IgG4 overexpression is also observed in other disorders, it is necessary to diagnose IgG4-related disease carefully and correctly. We therefore sought to determine cutoff values for serum IgG4 and IgG4/IgG and for IgG4+/IgG+ plasma cells in tissue diagnostic of IgG4-related disease. Patients and Methods. We retrospectively analyzed serum IgG4 concentrations and IgG4/IgG ratio and IgG4+/IgG+ plasma cell ratio in tissues of 132 patients with IgG4-related disease and 48 patients with other disorders. Result. Serum IgG4 $>135 \mathrm{mg} / \mathrm{dl}$ demonstrated a sensitivity of $97.0 \%$ and a specificity of $79.6 \%$ in diagnosing IgG4-related disease, and serum IgG4/IgG ratios $>8 \%$ had a sensitivity and specificity of $95.5 \%$ and $87.5 \%$, respectively. IgG4+cell/IgG+ cell ratio in tissues $>40 \%$ had a sensitivity and specificity of $94.4 \%$ and $85.7 \%$, respectively. However, the number of IgG4+ cells was reduced in severely fibrotic parts of tissues. Conclusion. Although a recent unanimous consensus of all relevant researchers in Japan recently established the diagnostic criteria for IgG4-related disease, findings such as ours indicate that further discussion is needed. 


\section{Introduction}

IgG4-related disease (IgG4-RD), a new disease classification first established in Japan in the 21st century, is characterized by hyper-IgG4-gammaglobulinemia and massive infiltration of IgG4-positive plasma cells into various swollen organs [1-10]. In general, a serum IgG4 concentration $>135 \mathrm{mg} / \mathrm{dL}$ has been established as the cutoff value for the diagnosis of patients with IgG4-RD and is used in the joint consensus criteria of the Okazaki and Umehara groups investigating IgG4-RD for the Ministry of Health, Labor, and Welfare of Japan [11].

Some patients with early or limited stage IgG4-RD, however, may show the sufficient pathological characteristics and clinical features of this disease, such as good response to glucocorticoids, despite having serum IgG4 concentrations $<135 \mathrm{mg} / \mathrm{dL}$. In addition, an IgG4 $+/ \mathrm{IgG}+$ ratio $>40 \%$ in tissue and $>10$ cells/high-power field (HPF) have been used in the histopathologic diagnosis of IgG4-RD in Japan. Thus, a proper diagnosis of these patients may require the use of other criteria, including IgG4+/IgG plasma cell ratio. Since lower cutoff values may increase sensitivity while decreasing specificity, it is necessary to establish accurate cut off values for this ratio.

To better establish the diagnostic criteria for IgG4-RD, we, the members of the IgG4+MOLPS/Mikulicz's disease research group in Japan, sought to determine the cutoff values for serum IgG4 and IgG4/IgG and for IgG4+/IgG+ plasma cells in tissue diagnostic of IgG4-RD using retrospectively collected data.

\section{Materials and Methods}

2.1. Measurement of Serum IgG4 Concentration. Serum IgG4 concentrations and IgG4/IgG ratio and the ratio of IgG4+/ IgG+ plasma cells in tissue were determined in 132 patients with IgG4-RD and 48 patients with other disorders registered retrospectively in the IgG4+MOLPS/Mikulicz's disease research group (Table 1). The 48 patients with other disorders included 33 with Sjögren's syndrome, 3 with multicentric Castleman's disease (MCD), 3 with B-cell lymphoma, 2 with sarcoidosis, and 1 each with Kimura's disease, ulcerative colitis, autoimmune hepatitis, IgG-type monoclonal gammopathy of undetermined significance, progressive transformation of the germinal center, scleritis, and severe keratoconjunctivitis sicca. The study was approved by the review boards of Kanazawa Medical University and all other collaborating institutions, and all patients provided written informed consent for the use of their data and samples. The sensitivities, specificities, and ROC curves of serum IgG4 $>135 \mathrm{mg} / \mathrm{dL}$ and various serum IgG4/IgG ratios were statistically analyzed using SPSS v.11 (SPSS Inc., Chicago, IL, USA).

Patients with borderline IgG4-RD were defined carefully as those with (1) $>40 \%$ IgG4+/IgG+ plasma cells in tissue, (2) strict pathological differential, and (3) a typical clinical
TABLE 1: Involved organs in 132 patients with IgG4-RD. More than half (64 cases) the patients in our series had so-called Mikulicz's disease, with symmetrical swelling of at least two sets of lacrimal, parotid, or submandibular glands.

\begin{tabular}{lc}
\hline Involved organ & Number of patients \\
\hline Total & 132 \\
\hline Lacrimal gland & 75 \\
Parotid gland & 32 \\
Submandibular gland & 74 \\
Pancreas & 20 \\
Kidney & 21 \\
Lung & 6 \\
Lymph node & 4 \\
Thyroid & 4 \\
Liver & 3 \\
Retroperitoneum & 3 \\
Dura & 1 \\
Hypophysis & 1 \\
Bile duct & 1
\end{tabular}

course (spontaneous regression or no change without treatment, or good response to an initial daily dose of $<0.6 \mathrm{mg} / \mathrm{kg}$ prednisolone).

2.2. Analysis of IgG4+ Cells in Tissue. The numbers of IgG4+ and IgG+ cells in tissue samples from 36 patients with IgG4$\mathrm{RD}$ and from 21 with other disorders were determined by counting cells counts in 5 high-power fields (HPF) under light microscopy. We also recounted cell areas of 17 samples from patients with IgG4-RD that contained both fibrotic and nonfibrotic parts.

We also assessed the sensitivity and specificity of IgG4+/ IgG+ cell ratios $>10 \%,>20 \%,>30 \%,>40 \%$, and $>50 \%$, and of $>10,>20,>30,>40$ and $>50$ IgG4+cells/HPF, as well as the presence of obliterative phlebitis, storiform fibrosis, eosinophilia, fibrosis, and lymphocyte infiltration as determined by hematoxylin and eosin staining in the diagnosis of IgG4-RD.

Tissue samples with borderline IgG4-RD were defined as those with (1) serum IgG4 $>135 \mathrm{mg} / \mathrm{dL}$, (2) strict pathological differential, and (3) typical clinical course. Clinical course including response to steroid is not included in the comprehensive diagnostic criteria for IgG4-RD, to avoid the needless treatment with steroid of patients suspected of having IgG4-RD. As this was a retrospective analysis, however, we analyzed the clinical course of these borderline patients, including their response to steroid treatment.

\section{Results}

3.1. Serum IgG4 Concentration. A serum IgG4 cutoff value $>135 \mathrm{mg} / \mathrm{dL}$ had a sensitivity of $97.0 \%$ and a specificity of $79.6 \%$ for the diagnosis of IgG4-RD. In 4 patients with relatively small and restricted lesions, however, this criterion was not adequate to diagnose IgG4-RD, although all had a 


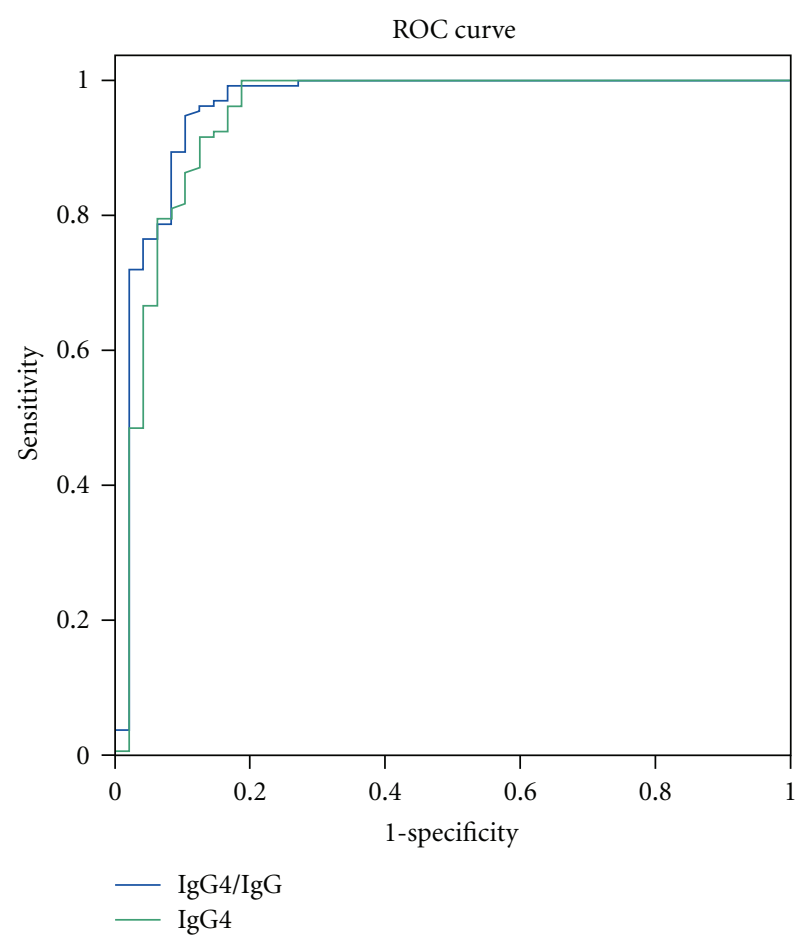

FIGURE 1: ROC curves of absolute serum IgG4 concentration (green) and serum IgG4/IgG ratio (blue). The two curves were almost identical.

histopathology and clinical course compatible with IgG4-RD (Table 2). All 4 patients were diagnosed with IgG4-RD based on a serum $\operatorname{IgG} 4 / \operatorname{IgG}$ ratio $>8 \%$.

In contrast, neither a serum IgG4 cutoff of $>135 \mathrm{mg} / \mathrm{dL}$ nor a serum IgG4/IgG ratio $>8 \%$ was adequate for the diagnosis of four patients with MCD and one each with Bcell lymphoma, scleritis, and Sjögren's syndrome, because all of these patients had hyper-IgG4-globulinemia associated with polyclonal gammopathy. We therefore estimated the sensitivity and specificity of various $\mathrm{IgG} 4 / \mathrm{IgG}$ ratios (Table 3). We found that the ROC curves for absolute serum IgG4 concentration and serum IgG4/IgG ratio were almost identical (Figure 1).

3.2. Analysis of IgG4+ Cells in Tissue Samples. We also assessed the ability of the ratio of IgG4+/IgG+ plasma cell ratios in $5 \mathrm{HPF}$ of tissue samples to diagnose IgG4-RD. We found that a ratio $>40 \%$ had a sensitivity of $94.4 \%$ and a specificity of $85.7 \%$ (Table 4 ). Although $>10$ IgG4+ cells per HPF had a sensitivity of $100 \%$, They had specificities of only $38.1 \%$.

In tissues containing both fibrotic and nonfibrotic areas, we counting the number cells in each part showed that fibrotic areas contained fewer IgG4+ cells (Table 5).

We also assessed the ability of obliterative phlebitis and storiform fibrosis to diagnose IgG4-RD. Although both had specificities of $100 \%$, their sensitivities were not very high (Table 4).
TABLe 2: Serum IgG and IgG4 concentrations and IgG4/IgG4 ratio of patients with false-positive and false-negative diagnoses of IgG4RD.

\begin{tabular}{lccc}
\hline False positives & & & \\
\hline Diagnosis & $\begin{array}{c}\text { IgG } \\
(\mathrm{mg} / \mathrm{dL})\end{array}$ & $\begin{array}{c}\text { IgG4 } \\
(\mathrm{mg} / \mathrm{dL})\end{array}$ & IgG4/IgG \\
\hline (1) MCD & 7,080 & 4,560 & $64.4 \%$ \\
(2) MCD & 4,420 & 690 & $15.6 \%$ \\
(3) B-cell lymphoma & 2,510 & 456 & $18.2 \%$ \\
(4) MCD & 2,960 & 295 & $10.0 \%$ \\
(5) MCD & 2,476 & 295 & $11.9 \%$ \\
(6) B-cell lymphoma & 4,010 & 271 & $6.8 \%$ \\
(7) Scleritis & 2,900 & 232 & $8.0 \%$ \\
(8) Sarcoidosis & 1,380 & 189 & $13.7 \%$ \\
(9) Sjögren's syndrome & 3,920 & 171 & $4.4 \%$ \\
(10) Malignant lymphoma & 1,930 & 141 & $7.3 \%$ \\
\hline False negatives & & & \\
\hline Diagnosis & IgG(mg/dL) & IgG4(mg/dL) & IgG4/IgG \\
\hline (1) IgG4-RD & 1,149 & 125 & $10.9 \%$ \\
(2) IgG4-RD & 1,210 & 123 & $10.2 \%$ \\
(3) IgG4-RD & 1,228 & 111 & $9.0 \%$ \\
(4) IgG4-RD & 1,260 & 106 & $8.4 \%$ \\
\hline
\end{tabular}

IgG4-RD: IgG4-related disease; MCD: multicentric Castleman's disease.

TABLe 3: Sensitivity and specificity of serum cutoff values in the diagnosis of IgG4-RD.

\begin{tabular}{lcc}
\hline & Sensitivity & Specificity \\
\hline IgG4 > 135 mg/dL & $97.0 \%$ & $79.6 \%$ \\
IgG4/IgG > 5\% & $99.2 \%$ & $83.3 \%$ \\
IgG4/IgG > 6\% & $97.0 \%$ & $83.3 \%$ \\
IgG4/IgG > 7\% & $97.0 \%$ & $85.4 \%$ \\
IgG4/IgG > 8\% & $95.5 \%$ & $87.5 \%$ \\
IgG4/IgG > 9\% & $92.4 \%$ & $89.6 \%$ \\
IgG4/IgG > 10\% & $89.4 \%$ & $91.7 \%$ \\
\hline
\end{tabular}

\section{Discussion}

Serum IgG4 $>135 \mathrm{mg} / \mathrm{dL}$ has been widely accepted as a cutoff value for diagnosis of IgG4-RD. Although this concentration was determined by comparing patients with IgG4-related sclerosing pancreatitis and those with pancreatic cancer [1], it has also been used to diagnose IgG4-RD involving other organs. For example, we have utilized this cutoff value as a diagnostic criterion for IgG4-related Mikulicz's disease [5] and IgG4-related kidney disease [12], and, in 2011, it was adopted in the comprehensive clinical diagnostic criteria of IgG4-related diseases [11]. Most patients with IgG4-RD show multiple organ involvement at diagnosis, with both high absolute serum IgG4 concentrations and serum IgG4/IgG ratios. However, some patients with early and/or limited stage IgG4-RD do not present with high IgG4-globulinemia (Figure 2), with some not having IgG4 concentrations $>135 \mathrm{mg} / \mathrm{dL}$. We have therefore tested the 
TABLE 4: Sensitivity and specificity of pathological findings for the diagnosis of IgG4-RD.

\begin{tabular}{lcc}
\hline & Sensitivity & Specificity \\
\hline IgG4+/IgG+ > 10\% & $100.0 \%$ & $33.3 \%$ \\
IgG4+/IgG+ > 20\% & $100.0 \%$ & $47.6 \%$ \\
IgG4+/IgG+ > 30\% & $100.0 \%$ & $71.4 \%$ \\
IgG4+/IgG+ > 40\% & $94.4 \%$ & $85.7 \%$ \\
IgG4+/IgG+ > 50\% & $94.4 \%$ & $95.2 \%$ \\
IgG4+cells/HPF > 10 & $100.0 \%$ & $38.1 \%$ \\
IgG4+cells/HPF > 20 & $97.2 \%$ & $42.9 \%$ \\
IgG4+cells/HPF > 30 & $97.2 \%$ & $61.9 \%$ \\
IgG4+cells/HPF > 40 & $91.7 \%$ & $66.7 \%$ \\
IgG4+cells/HPF > 50 & $86.1 \%$ & $71.4 \%$ \\
Obliterative phlebitis & $54.5 \%$ & $100.0 \%$ \\
Storiform fibrosis & $31.4 \%$ & $100.0 \%$ \\
Eosinophilia & $42.9 \%$ & $100.0 \%$ \\
Fibrosis & $91.4 \%$ & $82.4 \%$ \\
Lymphocytic & $100.0 \%$ & $16.7 \%$ \\
infiltration & &
\end{tabular}

TABLE 5: Recounting in each areas of 17 samples containing both fibrotic and nonfibrotic parts. All patients were diagnosed with IgG4-RD but had biopsy specimens that were too small (samples $1-14$ ) or with relatively large fibrotic areas inadequate to diagnose IgG4-RD (samples 15-17). All samples had >10 IgG4+ cells per HPF.

\begin{tabular}{lcc}
\hline Tissue & Fibrosis+ & Fibrosis- \\
\hline (1) Pancreas & $76.6 \%$ & $94.0 \%$ \\
(2) Submandibular gland & $75.4 \%$ & $89.2 \%$ \\
(3) Submandibular gland & $72.1 \%$ & $73.0 \%$ \\
(4) Submandibular gland & $72.1 \%$ & $97.3 \%$ \\
(5) Submandibular gland & $71.8 \%$ & $99.1 \%$ \\
(6) Pancreas & $67.7 \%$ & $95.0 \%$ \\
(7) Labial salivary glands & $65.0 \%$ & $70.8 \%$ \\
(8) Lung & $58.9 \%$ & $94.4 \%$ \\
(9) Submandibular gland & $49.2 \%$ & $68.9 \%$ \\
(10) Gall bladder & $48.6 \%$ & $94.0 \%$ \\
(11) Bile duct & $46.8 \%$ & $95.0 \%$ \\
(12) Submandibular gland & $46.2 \%$ & $74.1 \%$ \\
(13) Orbit & $44.2 \%$ & $94.4 \%$ \\
(14) Submandibular gland & $43.6 \%$ & $95.0 \%$ \\
(15) Submandibular gland & $33.3 \%$ & $95.0 \%$ \\
(16) Submandibular gland & $25.9 \%$ & $51.5 \%$ \\
(17) Labial salivary glands & $8.0 \%$ & $76.2 \%$ \\
\hline
\end{tabular}

ability of alternative criteria to diagnose for IgG4-RD. Although we found that a serum IgG4/IgG ratio $>5 \%$ had the highest sensitivity, the normal ratio is about $5-6 \%$, making this cut off value misleading. An IgG4/IgG ratio $>8 \%$ had a sensitivity similar to that of absolute IgG4 $>135 \mathrm{mg} / \mathrm{dL}$, but a greater specificity, enabling us to diagnose 4 patients with lower absolute IgG4 concentrations as having



Figure 2: Histopathology of a patient with limited stage IgG4related dacryoadenitis. A 35-year-old woman presented with swelling of her bilateral lacrimal glands. Her serum IgG and IgG4 concentrations were $1,149 \mathrm{mg} / \mathrm{dL}$ and $125 \mathrm{mg} / \mathrm{dL}$, respectively, and her serum IgG4/IgG ratio was $10.88 \%$. Histopathological examination of a lacrimal gland biopsy revealed IgG4+ plasma cell infiltration, with an IgG4+/IgG+ cell ratio of $74.7 \%$, and 53 IgG4+ cells per HPF, although sclerotic changes were not severe. Treatment with prednisolone $20 \mathrm{mg}$ /day resulted in a rapid and dramatic improvement in symptoms. Although using an absolute serum IgG4 cutoff concentration of $>135 \mathrm{mg}$ did not result in diagnosis of IgG4$\mathrm{RD}$, her clinical course and histopathology were typical of IgG4RD. However, using a serum $\operatorname{IgG} 4 / \operatorname{IgG}$ ratio $>8 \%$ as a cutoff value resulted in a diagnosis of IgG4-RD.

IgG4-RD (Table 2). Since the standard cut off of absolute IgG4 >135 mg/dL demonstrated excellent sensitivity and specificity, it should be utilized, except for patients with early and/or limited IgG4-RD, for whom we propose using an IgG4/IgG ratio $>8 \%$.

Careful diagnosis is required in patients with lower IgG4 concentrations, since those patients may have other distinct disorders with different clinical features than IgG4RD. Patients with untypical clinical courses, including glucocorticoid refractoriness, should be reassessed.

IgG4+/IgG+ plasma cell ratios in tissue $>40 \%$ and $>50 \%$, and $>10$ IgG4+ cells per HPF have been used for the diagnosis of IgG4-RD. We found that an IgG4+/IgG+ cell ratio $>40 \%$ in tissue had a sensitivity of $94.4 \%$ and a specificity of $85.7 \%$ in the diagnosis of IgG4-RD. We also found that IgG4+ plasma cell concentrations in tissue were diminished in fibrotic tissue areas, suggesting that a ratio $>40 \%$ is a better histopathologic cutoff value. The presence of obliterative phlebitis and storiform fibrosis demonstrated specificities of $100 \%$, but their sensitivities were much lower, indicating that these findings would be useful when added to, but not in place of, other results.

Since patients with disorders such as MCD and lymphoma may demonstrate hyper-IgG4-gammaglobulinemia and massive IgG4+ plasma cell infiltration in tissue, serum IgG4 concentration and IgG4+ cells in tissue are not specific indicators of IgG4-RD. Rather, a diagnosis of IgG4-RD should be based on the overall balance of clinical features, such as disease distribution throughout the body, clinical course, serum concentrations, and histopathology.

The pathologic consensus statement of the first international Symposium on IgG4-RD in Boston did not adopt IgG4+/IgG+ cell ratio in tissue as diagnostic, although it 
did suggest cutoffs for numbers of IgG4+ cells in HPFs of various organs. This, however, may be confusing for many pathologists and physicians. Although pathologic findings are very important in the diagnosis for IgG4-RD, clinical features and serological findings should be included.

Recently, some patients with IgG4-RD were found to have lymphoma $[13,14]$ and other types of cancer $[15,16]$. Thus IgG4-RD may not always be a benign disease with good prognosis. Many patients referred to our centers with glucocorticoid refractory IgG4-RD were diagnosed incorrectly, suggesting the need for more accurate diagnostic criteria for these diseases.

\section{Acknowledgments}

The authors thank all participants in the All Japan, Ministry of Health, Labor, and Welfare (MHLW) IgG4 team for critical discussion. Funding: This paper was partially supported by the Research Program of Intractable Disease provided by the Ministry of Health, Labor, and Welfare (MHLW) of Japan, and by the Japanese Ministry of Education, Culture, Sports, Science and Technology (Grant nos. 13557160, 15024236,15390313 , and 13877075 to $H$. Umehara and no. 17591060 to Y. Masaki). It was also supporrted by the Uehara Memorial Foundation (to H. Umehara), the Vehicle Racing Commemorative Foundation, the Kanazawa Medical University Research Foundation (Grant nos. C2009-4 to Umehara and S2004-16 and S2007-5 to Y. Masaki), Grant for Assist KAKEN from Kanazawa Medical University (Grant no. K2011-7), and Grant for Project Research from High-Tech Research Center of Kanazawa Medical University (Grant no. H2011-11).

\section{References}

[1] H. Hamano, S. Kawa, A. Horiuchi et al., "High serum IgG4 concentrations in patients with sclerosing pancreatitis," New England Journal of Medicine, vol. 344, no. 10, pp. 732-738, 2001.

[2] T. Kamisawa and A. Okamoto, "Autoimmune pancreatitis: Proposal of IgG4-related sclerosing disease," Journal of Gastroenterology, vol. 41, no. 7, pp. 613-625, 2006.

[3] Y. Masaki, L. Dong, N. Kurose et al., "Proposal for a new clinical entity, IgG4-positive multiorgan lymphoproliferative syndrome: analysis of 64 cases of IgG4-related disorders," Annals of the Rheumatic Diseases, vol. 68, no. 8, pp. 1310-1315, 2009.

[4] M. Yamamoto, H. Takahashi, K. Hasebe et al., "The analysis of interleukin-6 in patients with systemic IgG4-related plasmacytic syndrome-expansion of SIPS to the territory of Castleman's disease," Rheumatology, vol. 48, no. 7, pp. 860862, 2009.

[5] Y. Masaki, S. Sugai, and H. Umehara, "IgG4-related diseases including Mikulicz's disease and sclerosing pancreatitis: diagnostic insights," Journal of Rheumatology, vol. 37, no. 7, pp. 1380-1385, 2010.

[6] Y. Masaki, H. Iwao, A. Nakajima, M. Miki, S. Sugai, and H. Umehara, "IgG4-related disease (IgG4+MOLPS)—diagnostic criteria and diagnostic problems," Current Immunology Reviews, vol. 7, no. 2, pp. 172-177, 2011.
[7] Y. Masaki, N. Kurose, and H. Umehara, "IgG4-related disease: a novel lymphoproliferative disorder discovered and established in Japan in the 21st century," Journal of Clinical and Experimental Hematopathology, vol. 51, pp. 13-20, 2010.

[8] T. Saeki, S. Nishi, N. Imai et al., "Clinicopathological characteristics of patients with IgG4-related tubulointerstitial nephritis," Kidney International, vol. 78, no. 10, pp. 10161023, 2010.

[9] Y. Sato, K. Notohara, M. Kojima, K. Takata, Y. Masaki, and T. Yoshino, "IgG4-related disease: Historical overview and pathology of hematological disorders," Pathology International, vol. 60, no. 4, pp. 247-258, 2010.

[10] H. Umehara, K. Okazaki, Y. Masaki et al., " The Research Program for Intractable Disease by Ministry of Health, Labor and Welfare (MHLW) Japan G4 team. A novel clinical entity, IgG4-related disease (IgG4RD): general concept and details," Modern Rheumatology, vol. 22, pp. 1-14, 2012.

[11] H. Umehara, K. Okazaki, Y. Masaki et al., "Comprehensive diagnostic criteria (CDC) for IgG4-related disease (IgG4RD) 2011," Modern Rheumatology, vol. 22, pp. 21-30, 2012.

[12] M. Kawano, T. Saeki, H. Nakashima et al., "Proposal for diagnostic criteria for IgG4-related kidney disease," Clinical and Experimental Nephrology, vol. 15, pp. 615-626, 2011.

[13] W. Cheuk, H. K. L. Yuen, A. C. L. Chan et al., "Ocular adnexal lymphoma associated with IgG4+ chronic sclerosing dacryoadenitis: a previously undescribed complication of IgG4-related sclerosing disease," American Journal of Surgical Pathology, vol. 32, no. 8, pp. 1159-1167, 2008.

[14] Y. Sato, K. I. Ohshima, K. Ichimura et al., "Ocular adnexal IgG4-related disease has uniform clinicopathology," Pathology International, vol. 58, no. 8, pp. 465-470, 2008.

[15] O. Hyoung-Chul, K. J. Gyu, K. J. Wook et al., "Early bile duct cancer in a background of sclerosing cholangitis and autoimmune pancreatitis," Internal Medicine, vol. 47, no. 23, pp. 2025-2028, 2008.

[16] T. Kamisawa, P. Y. Chen, Y. Tu et al., "Pancreatic cancer with a high serum IgG4 concentration," World Journal of Gastroenterology, vol. 12, no. 38, pp. 6225-6228, 2006. 




The Scientific World Journal
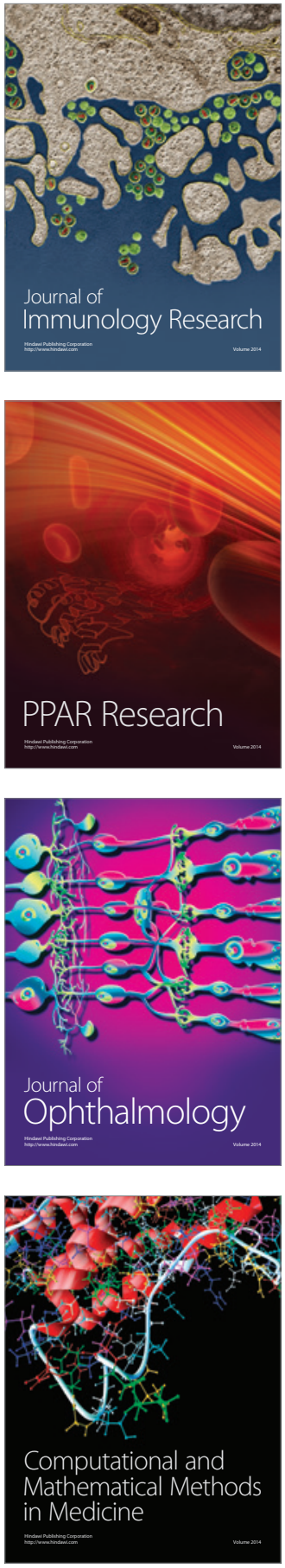

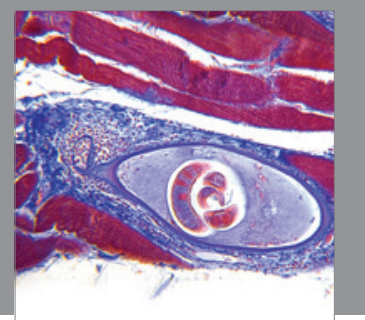

Gastroenterology

Research and Practice
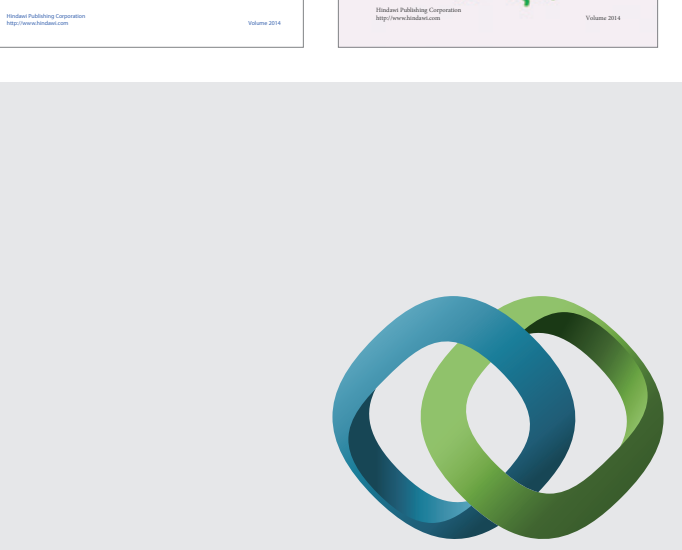

\section{Hindawi}

Submit your manuscripts at

http://www.hindawi.com
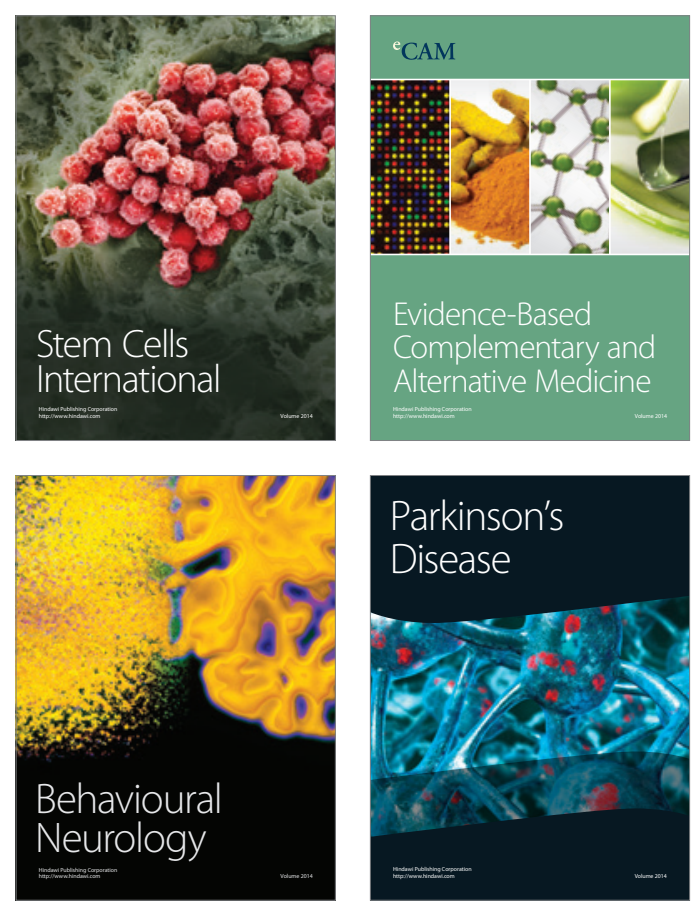

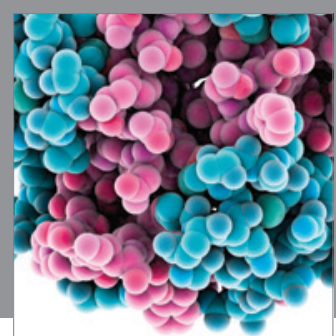

Journal of
Diabetes Research

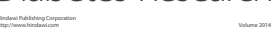

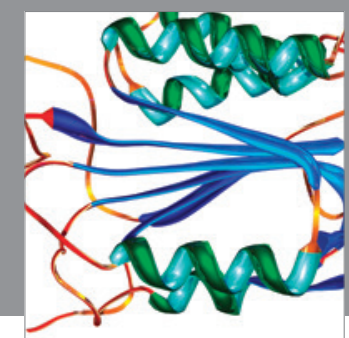

Disease Markers
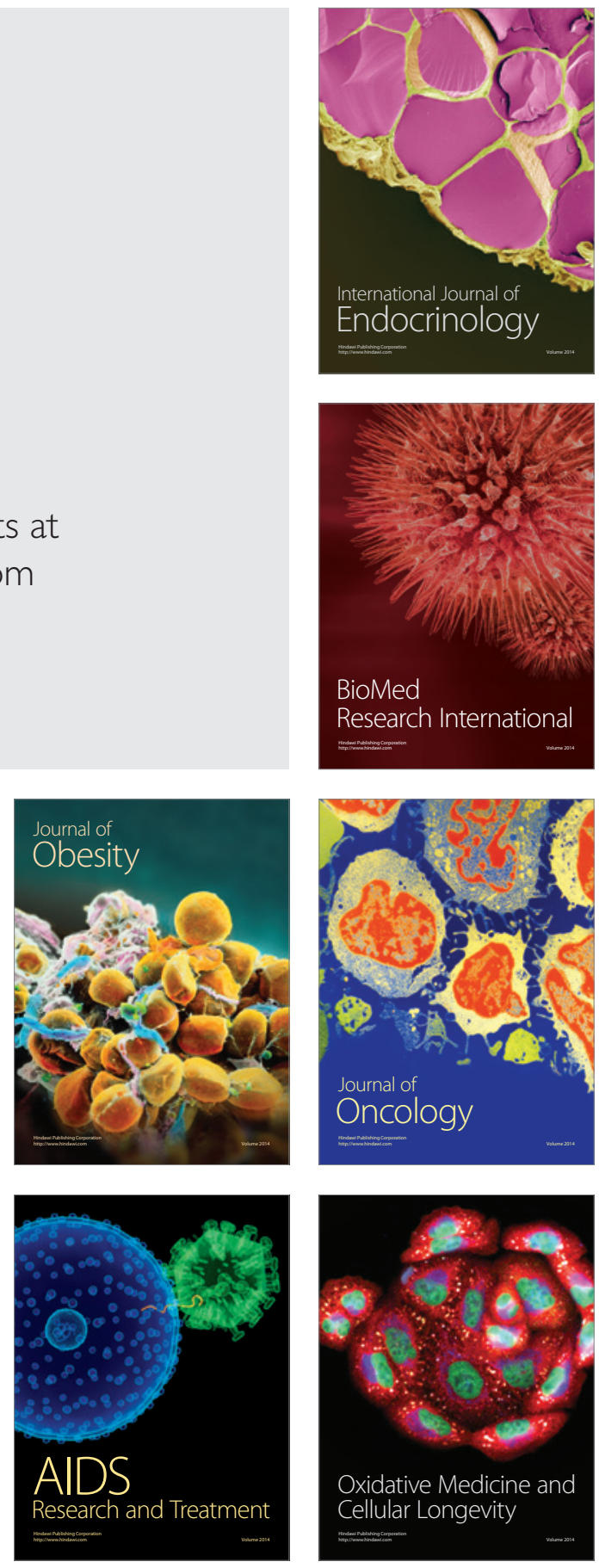